\title{
Radio burst statistics for $M$ dwarf flare stars
}

\author{
M. Abada-Simon ${ }^{1}$ and M. Aubier ${ }^{1,2}$ \\ 1 ARPEGES/URA CNRS 1757, Observatoire de Paris, 92190 Meudon, France \\ ${ }^{2}$ Université de Versailles Saint-Quentin-en-Yvelines, France
}

Received March 8; accepted February 12, 1997

\begin{abstract}
We present a critical analysis on the number of bursts near $21 \mathrm{~cm}$ from nine dMe flare stars of the solar neighborhood as reported in the literature. We compare our findings with the number of bursts detected with the Arecibo radiotelescope between 1989 and 1993, using a very reliable method to discriminate between stellar and artificial emissions. We compare the rates of radio bursts per hour inferred from both this Arecibo campaign and the literature to rates of optical flares found in the literature. We also compare the nine stars to each other, and the Arecibo results to radio surveys of two open clusters.
\end{abstract}

Key words: stars: flare; late type; activity — radio continum: stars - methods: statistical

\section{Introduction}

dMe flare stars are main sequence stars of spectral type $\mathrm{M}$, exhibiting hydrogen and ionised calcium emission lines. They are smaller and less massive than the Sun, and their rotation period is of the order of days. They are known to be very active stars, with flare energy releases reaching up to $10^{3}$ times those of the Sun in the visible and radio domains (Haisch 1989). It has been suggested that some dMe stars may exhibit cyclic variations with a period of the order of 10 years (e.g. Phillips \& Hartmann 1978; Pettersen et al. 1986; Jetsu 1993; Bondar 1995), and it has been estimated that some dMe stars exhibit about 0.2 to 1.5 visible flares per hour. However, their rate of activity in radio, defined as the number of observed radio bursts per hour, is not well established, and this is a useful parameter to understand the physical processes occurring in a stellar atmosphere.

The numerous publications reporting radio bursts date to the early sixties and might have given the impression that bursts from dMe flare stars could be "easily" detected. However, one can follow, through these publications, that various observing techniques were used and improved to try to distinguish between artificial radiofrequency interference and bursts of stellar origin. This suggests that with the techniques used in the past, some artificial events might have been taken for bursts of stellar origin.

In Sect. 2, we present our critical analysis of the bursts near $21 \mathrm{~cm}$ published in the past, and we derive estimates on the rate of bursts one may expect. In Sect. 3, we give the rates inferred from a 200 hour observing campaign performed between 1989 and 1993, using a very reliable technique on the most sensitive single-dish radiotelescope, located in Arecibo (Puerto-Rico). In both Sects. 2 and 3, we focus our study on the bursts detected near $21 \mathrm{~cm}$ from the nine dMe flare stars observed during this campaign: AD Leonis, YZ Canis Minoris, YY Gemminorum, EQ Pegasi, TZ Arietis, Wolf 424, Gliese 569, V371 Orionis, and VW Coma Berenices. Some of their properties are recalled in Table 2. In Sect. 4, we compare the rates predicted from the literature with the rates found with the Arecibo campaign, and we compare them to radio surveys of open clusters and to optical flare rates. In Sect. 5, we summarize our results and conclude.

\section{Flaring activity established from previous publications}

\subsection{Selection criteria}

For each of the nine dMe flare stars listed above, we have counted the number of bursts reported in the literature, taking into account the methods used to prove the stellar origin of the bursts and the total number of observing hours. Many radio bursts were detected in the metre range, but we have focussed on the observations near $21 \mathrm{~cm}$ in order to have data comparable to our observations. On the other hand, an important part of bursts was detected at $6 \mathrm{~cm}$ (mainly with the Very Large Array VLA), and many of them are reported in the same papers as for the bursts near $21 \mathrm{~cm}$ (see for example the large survey by White et al. 1989). In particular, very weak detections (about $1 \mathrm{mJy}$ ) with the VLA have been reported in the publications. All these reports might have contributed to the impression that radio bursts could easily be detected from flare stars. 
Assuming that the activity of the nine studied stars has not changed since they are observed at decimetric wavelengths, the burst rates inferred from the literature should be the same as the rates deduced from the reliable technique used between 1989 and 1993 (Lecacheux et al. 1993; Abada-Simon et al. 1994), under the two following conditions on the bursts reported in the publications: firstly, we only take into account the bursts for which the stellar origin is proven; secondly, we take into account only the bursts reaching at least $8 \mathrm{mJy}$, which is the peak flux density of the weakest burst detected during the Arecibo campaign.

We have considered that the stellar origin of a burst is proven when one of the three following techniques is used: i) use of an interferometer (mainly the VLA): this kind of instrument allows to create a map of the observed star and the surrounding sky region, and only an emission recorded by all the antennas can produce a "consistent" signal appearing at the position of the star. In addition, a map is useful in identifying sources which are close to the observed star and which are known to radiate at $21 \mathrm{~cm}$ too: this prevents from the source confusion problem of singledish telescopes; for example, there is a well-known extragalactic source detected in a direction close to that of $\mathrm{AD}$ Leo: the emission of T1017+201 (flux density of $200 \mathrm{mJy}$, e.g. Jackson et al. 1989) is within the primary beam of the Arecibo telescope, and occasionally needs to be distinguished from a possible stellar burst;

ii) recording simultaneously a burst with several (singledish) radiotelescopes observing all near $21 \mathrm{~cm}$;

iii) observing simultaneously the star ("ON") and another close direction in the sky ("OFF") with two antennas (mostly single-channel) tuned at almost equal frequencies (near $21 \mathrm{~cm}$ ).

We have not taken into account the bursts detected by a single-dish telescope for which the stellar origin was not proven by one of the above criteria, especially when a burst is recorded with a single- frequency channel receiver. However, we cannot reject some bursts as definitely not stellar. In particular, one can find apparent rapid "spikes", without being able to prove their stellar origin. Such "false" spikes were discovered in the data during the campaign of 1989-1993 in Arecibo (Lecacheux et al. 1992). Finally, the (quasi-)simultaneity of a radio event with a flare at other wavelengths may not be a sufficient proof of its stellar origin.

\subsection{Difficulties encountered in the literature}

One problem is that the precise number of bursts detected is not always specified by the authors, and another one is that the total number of observing hours is not always given. We report such incomplete data but we cannot use them to estimate the rate of activity in radio. In addition, the observing techniques used in the past may have had different sensitivity thresholds, so that the weakest bursts were probably missed. The different time resolutions used on various instruments such as the VLA and the Arecibo telescope also suggest to be careful in comparing the bursts' fluxes. Furthermore, in the past, only the most intense bursts were reported, whereas weaker bursts might have been present in the data without being searched for (private communication). Some real bursts may also have been rejected because they didnot exhibit the typical characteristics expected from a star (e.g. strong polarisation, drift of the emission frequency with time). Finally, some detections of weak bursts were never reported in the literature after many days of observation (e.g. Bastian, private communication). All these facts may lead us to underestimate the rate of radio bursts per hour. On the other hand, it may be overestimated by the fact that some nondetections following many hours of observation were never reported. Finally, we might have missed some publications on the subject.

Another difficulty - that we also encountered during the observations of 1989-1993 in Arecibo - rests in counting the "real" bursts. There is no "strict" distinction between a quiescent emission (i.e. weak and slowly varying) and a burst (stronger and more rapid). Indeed, quiescent emission from UV Ceti, the prototype of dMe stars, was recorded up to $18 \mathrm{mJy}$ (Bastian \& Bookbinder 1987), and this value is higher than many bursts. On the other hand, YZ CMi is a good example of a dMe star exhibiting gradual increases and decreases which can hardly be classified as quiescence or burst: how can one estimate the number of individual bursts in a "weakly varying event" of YZ CMi, as decreasing from 12 to 6 mJy during 5 hours (see Kundu et al. 1986)? Another difficulty is then to distinguish several "independent" bursts which are close in time from several bursts which actually belong to the same "single event".

\subsection{Results from the publications}

In the tables of Appendix 1, we present our findings for eight of the nine dMe stars listed in Sect. 1 (we could not find any report on a detection of V371 Ori near 21 $\mathrm{cm})$. One can see in Appendix 1 a case when AD Leo was observed simultaneously at four frequencies by the VLA: a burst reached $80 \mathrm{mJy}$ at $1415 \mathrm{MHz}$ but it was not detected at $1515 \mathrm{MHz}$, nor at $5 \mathrm{GHz}$ (Kundu et al. 1986). Since a reliable observing technique was used, this result suggests that the bandwidth of the burst did not extend to frequencies higher than $1415 \mathrm{MHz}$, but it may extend to lower frequencies.

In summary, AD Leo emitted 6 bursts in excess of $8 \mathrm{mJy}$ in 31 hours, EQ Peg more than 5 in $16 \mathrm{hr}$, and YZ CMi more than 13 in $34 \mathrm{hr}$; Wolf 424 and YY Gem exhibited no burst in 14 and 15 hr (respectively), nor did TZ Ari, Gl. 569 and VW Com (in only $0.4 \mathrm{hr}$ ). Let us see if the observations of 1989-1993 are in agreement with the preceding publications. 


\section{Activity revealed by the observations of 1989- 1993 in Arecibo}

\subsection{Reliability of the observing technique}

Knowing that narrow-band stellar bursts $(\Delta f / f \approx 1 \%)$ exist, the tuning frequencies of several antennas observing simultaneously should be spaced by less than about $10 \mathrm{MHz}$, otherwise the burst may be missed by one of the antennas. In addition, a day-to-day comparison of the data is especially useful in rejecting non-stellar emissions recorded by the antenna "ON". Therefore, when observing with a single-dish radiotelescope, the most reliable observations combine simultaneous $\mathrm{ON}$ and $\mathrm{OFF}$-source antennas, tuned at the same frequency, both of wide bandwidth, high frequency and time resolutions, and daily comparison of the data recorded at the same hour angle. This is the technique used during a 200 hour observing campaign with an acousto-optical spectrograph as a receiver of the Arecibo telescope. In the course of this campaign, which started in July 1989 and ended in February 1993, nine dMe flare stars and one binary system of the type RS Canum Venaticorum (RS CVn) were observed at 6 and 21 cm (Lecacheux et al. 1993; Abada-Simon et al. 1994).

\subsection{Number of bursts detected}

Only AD Leo was detected during the Arecibo campaign, and no burst was detected from the other eight dMe flare stars observed, nor from the RS CVn system UX Ari. The details on each of the eleven bursts detected from AD Leo in 38.3 hours of data are recalled in Table 1 (from Abada-Simon et al. 1994). In summary, half of the bursts is circularly polarised, half is not; the bursts last between 6 and $90 \mathrm{~s}$, and their peak flux density is between 8 and $63 \mathrm{mJy}$ with an integration time of $1 \mathrm{~s}$, but the strongest burst was in fact made of spikes reaching up to $350 \mathrm{mJy}$ in $20 \mathrm{~ms}$. Figure 1 shows a histogram of the eleven bursts' flux density. We have counted as "one individual burst" either an event isolated in a 5-min scan, or a burst separated from another one by a "non-detection time" lasting at least ten seconds: in this second case, it is possible that several "bursts" actually belong to the same event whose flux decreases below the detection threshold. The situation is different in the case of the event (counted as two bursts) recorded on 13 February 1993: the second burst is made of several brief bursts present over several minutes.

It is also interesting to note that the eleven bursts were detected in only a few days: two bursts on 11 December 1990 separated by less than 30 minutes, six bursts on 13 December 1990, among which five occurred over $15 \mathrm{~min}$ maybe a unique event? -, one burst on 12 February 1993, and 7 minutes of activity on 13 February 1993. The six bursts recorded on 13 December 1990 cover a period of time $\Delta t=1 \mathrm{~h} 15 \mathrm{~min}$, during which $\mathrm{AD}$ Leo rotates by an angle:

$\alpha(\mathrm{rad})=2 \pi \Delta t \sin (i) / P_{\text {rot }} \approx 4^{\circ}$

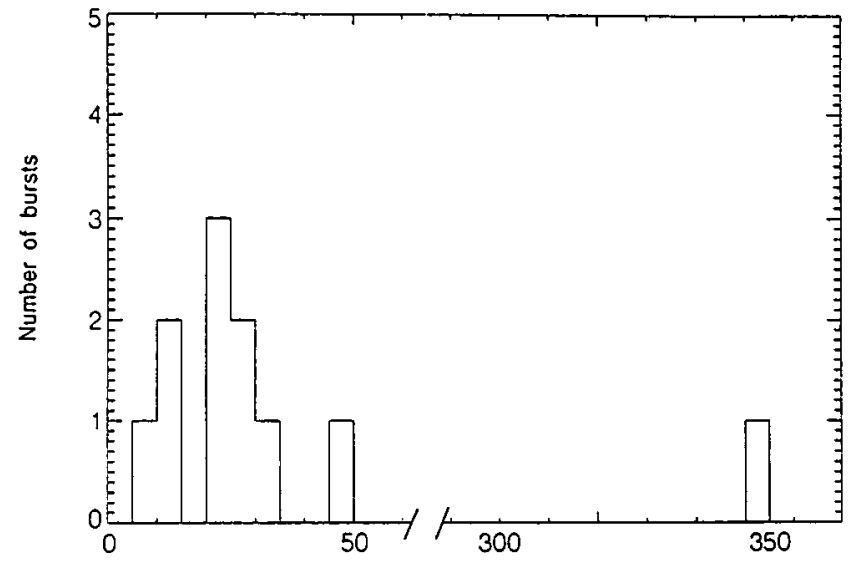

Fig. 1. Histogram of the bursts' peak flux density detected from AD Leo at $21 \mathrm{~cm}$ between 1990 and 1993 in Arecibo by Abada-Simon et al. (1994)

where $P_{\text {rot }}=2.7$ days is AD Leo's rotation period, and $i=$ $38^{\circ}$ is the inclination of its rotation axis to the line of sight (Pettersen et al. 1984). Assuming that these bursts are emitted from one "active region" and that the radiation is beamed toward the star's zenith, the size of this region is:

$L=\Delta t R \sin (i) / P_{\text {rot }} \approx 4000 \mathrm{~km}$

where $R$ is AD Leo's radius. The size $L$ may be smaller if the emitting region is not on the equator, but since radio bursts arise in the corona, i.e. up to about one stellar radius above the surface, the size $L$ of the "active region" may be underestimated by a factor two. Within the same assumptions of beamed radiation toward zenith and of one "unique active region" for the consecutive radio bursts, it is interesting to note that, since AD Leo's rotation period is 2.7 days, the radio bursts detected on 13 December 1990 could come from the same region as that from which the radio bursts of 11 December 1990 originated, after the "active region" was not observable from the Earth on 12 December 1990; the bursts detected on 12 and 13 February 1993 could also come from one region (observable on both days). There could be several reasons why radio emission is not detected continuously when the hypothetical "active region" is observable from the Earth: i) the emission of radio bursts might be triggered only from time to time in the "active region"; ii) the radio emission may be below the detection threshold during part of the observing time; iii) the radio bursts are probably emitted in a narrow solid angle and toward directions which vary with time. Knowing that these bursts emission processes are coherent, the latter possibility (iii) seems more realistic than a radio radiation beamed toward zenith.

Finally, in addition to the nine dMe flare stars, the RS CVn-type UX Arietis was also observed but no burst was detected during the campaign of 1989-1993 in Arecibo. In 
Table 1. Main characteristics of the bursts observed from AD Leo at $21 \mathrm{~cm}$ with the Arecibo radiotelescope (from Abada-Simon et al. 1994)

\begin{tabular}{lcccccc}
\hline Date & $\begin{array}{c}\text { Starting } \\
\text { Time (UT) }\end{array}$ & Duration (s) & $\begin{array}{c}\text { Peak Flux } \\
\text { Density in } \\
\text { RCP }(\text { mJy) }\end{array}$ & $\begin{array}{c}\text { Peak Flux } \\
\text { Density in } \\
\text { LCP }(\mathrm{mJy})\end{array}$ & RCP (\%) & LCP (\%) \\
\hline 11 Dec. 90 & $08: 41: 12$ & $\sim 12$ & $\sim 20(9.1 \sigma)$ & 0 & 100 & 0 \\
11 Dec. 90 & $09: 05: 10$ & $\sim 12$ & $\sim 8(5.7 \sigma)$ & 0 & 100 & 0 \\
13 Dec. 90 & $08: 37: 55$ & $\sim 6$ & $\sim 13(4.8 \sigma)$ & $\sim 12(4.3 \sigma)$ & 50 & 50 \\
13 Dec. 90 & $09: 40: 28$ & $\sim 15$ & $\sim 12(5.0 \sigma)$ & $\sim 13(4.8 \sigma)$ & 50 & 50 \\
13 Dec. 90 & $09: 41: 08$ & $\sim 14$ & $\sim 12(5.0 \sigma)$ & $\sim 12(4.4 \sigma)$ & 50 & 50 \\
13 Dec. 90 & $09: 42: 19$ & $\sim 41$ & $\sim 20(8.3 \sigma)$ & $\sim 26(9.6 \sigma)$ & 43 & 57 \\
13 Dec. 90 & $09: 51: 15$ & $\sim 46$ & $\sim 12(6 \sigma)$ & $\sim 12(6.7 \sigma)$ & 50 & 50 \\
13 Dec. 90 & $09: 53: 03$ & $\sim 10$ & $\sim 8(4 \sigma)$ & $\sim 6(3.3 \sigma)$ & 57 & 43 \\
12 Feb. 93 & $04: 40: 55$ & $\sim 8$ & 0 & $\sim 10(4.0 \sigma)$ & 0 & 100 \\
13 Feb. 93 & $04: 12: 53$ & $\sim 90$ & $\sim 63(12.5 \sigma)$ & 0 & 100 & 0 \\
13 Feb. 93 & $04: 17: 22$ & $\sim 40$ & $\sim 30(9.0 \sigma)$ & 0 & 100 & 0 \\
\hline
\end{tabular}

fact, UX Ari's flares are generally on a timescale of hours to days, as it has been observed by the VLA, but it can emit relatively short bursts. Its flux density at $5 \mathrm{GHz}$ can increase from 16 to $21 \mathrm{mJy}$ in about $10 \mathrm{~min}$ and decrease again to $16 \mathrm{mJy}$ in $10 \mathrm{~min}$ (Lefèvre et al. 1993): this kind of variability is too low and too slow to be easily identified with the Arecibo radiotelescope. Since UX Ari could not be tracked more than $1 \mathrm{~h} 15 \mathrm{~min}$ (at $21 \mathrm{~cm}$ ) with this instrument, its long duration flares cannot be observed; our result is that it emitted no short burst in $2.8 \mathrm{hr}$ of observation.

\subsection{Comparison between the dMe stars observed in Arecibo}

Before comparing the flare occurrence rates deduced from our observations to those found in the literature, let us see if the non-detection of eight stars among the nine dMe stars observed in Arecibo in 1989-1993 is surprising, assuming that their behaviour is totally comparable to that observed on AD Leo. Table 2 shows the number of observing hours (Col. 4) of each star (name in Col. 1) whose spectral type and distance are recalled in Cols. 2 and 3 (resp.). Columns 5, 6, 7 and 8 indicate the probability to detect $0,1,2$ and 3 bursts (respectively), under the following conditions. Eleven bursts were detected from AD Leo in $38.3 \mathrm{hr}$, and we assume that:

- the nine studied stars emit bursts of equal strength, - the other eight stars have the same flare rate occurrence as $\mathrm{AD}$ Leo,

- they are observed at the distance of AD Leo (4.9 pc), and

- their probability of burst emission follows the Poisson statistics law.
According to Poisson's law, the probability that $n$ bursts are emitted is:

$P(n)=\left(a^{n} \mathrm{e}^{-a}\right) / n !$

where $a$ is a parameter describing the distribution of bursts. For a star observed for $N$ hours, this parameter is, in the case of 12 bursts emitted by AD Leo in $38.3 \mathrm{hr}$ :

$a=11 N / 38.3$.

As an additional information, Cols. 9 and 10 indicate, for each star "placed at its real distance", the corresponding flux density of a burst of 8 and 64 mJy (respectively) when observed at $4.9 \mathrm{pc}$.

In the frame of the former hypotheses, we can see that, during the observations of 1989-1993, we had a maximum probability to detect no burst from VW Com, V371 Ori and YY Gem; we had a maximum probability to detect one burst from Gl 569; we had approximately equal probabilities to detect zero or one burst from EQ Peg and TZ Ari, one or two bursts from YZ CMi, and two or three bursts from Wolf 424: for these two latter stars, the probability to detect no burst was relatively low. However, apart from Wolf 424 and TZ Ari, the six other stars are further than AD Leo.

If we consider that the weakest burst from AD Leo (peak flux density of $8 \mathrm{mJy}$ ) is the weakest detectable at $21 \mathrm{~cm}$ by the Arecibo telescope (with the observing technique used in 1989-1993), it is obvious that if the six stars which are further than AD Leo emitted such a low burst during the observations of 1989-1993, it could not be detected. Among these six stars, only YZ CMi, which was observed for more than twice the time of the five others, could have emitted a second burst, but if it were as weak as the "second weaker" burst of AD Leo (10 mJy, that is $6.6 \mathrm{mJy}$ at YZ CMi's distance), we might not have detected it either. We have just taken the most pessimistic 
Table 2. Comparisons between the nine dMe stars observed, assuming they all behave as AD Leo

\begin{tabular}{|c|c|c|c|c|c|c|c|c|c|}
\hline Star & $\begin{array}{c}\text { Spec- } \\
\text { tral } \\
\text { Type }\end{array}$ & $\begin{array}{c}\text { Dis- } \\
\text { tance } \\
(\mathrm{pc})\end{array}$ & $\begin{array}{c}\text { Number } \\
\text { of } \\
\text { observin } \\
\text { g hours } \\
\text { at } 1.4 \\
\mathrm{GHz}\end{array}$ & $\begin{array}{c}P(0) \\
(\%)\end{array}$ & $\begin{array}{c}P(1) \\
(\%)\end{array}$ & $\begin{array}{c}P(2) \\
(\%)\end{array}$ & $\begin{array}{c}P(3) \\
(\%)\end{array}$ & $\begin{array}{c}\text { Flux } \\
\text { dens. at } \\
\text { diff. dist. } \\
\text { of a burst } \\
\text { of } 8 \text { mJy } \\
\text { at } 4.9 \text { pc }\end{array}$ & $\begin{array}{c}\text { Flux } \\
\text { dens. at } \\
\text { diff. dist. } \\
\text { of a burst } \\
\text { of } 64 \\
\text { mJy at } \\
4.9 \mathrm{pc}\end{array}$ \\
\hline Wolf 424 & M5.5Ve & 4.3 & 10.7 & 4.6 & 14.2 & 21.9 & 22.4 & 10.4 & 83.2 \\
\hline TZ Ari & M5Ve & 4.5 & 3.3 & 36.7 & 34.8 & 16.5 & 5.2 & 9.5 & 76 \\
\hline AD Leo & M4Ve & 4.9 & 38.3 & $<2.10^{-5}$ & $<2.10^{-4}$ & 0.1 & 0.4 & 8 & 64 \\
\hline YZ CMi & M4.5Ve & 6.0 & 7.7 & 11.0 & 24.2 & 26.8 & 19.7 & 5.3 & 42.4 \\
\hline EQ Peg & M4Ve & 6.5 & 3.6 & 35.6 & 36.8 & 19.0 & 6.6 & 4.5 & 36 \\
\hline Gl 569 & M6Ve & 10.4 & 4.4 & 28.3 & 35.7 & 22.6 & 9.5 & 1.8 & 14.4 \\
\hline YY Gem & M1Ve & 14.5 & 3.1 & 41.1 & 36.5 & 16.3 & 4.8 & 0.9 & 7.2 \\
\hline V371 Ori & M3Ve & 15.2 & 2.9 & 43.5 & 36.2 & 15.1 & 4.2 & 0.8 & 6.4 \\
\hline VW Com & M4Ve & 17.5 & 2.0 & 57.4 & 33.0 & 9.5 & 1.8 & 0.6 & 4.8 \\
\hline
\end{tabular}

hypotheses, assuming that the stars would have emitted the weakest bursts of AD Leo.

In summary, if the nine observed dMe stars were comparable, we could not be surprised by having detected no burst from TZ Ari and the six stars further than AD Leo, but we may be more surprised by knowing that bursts much stronger than the weakest ones assumed can be emitted. On the other hand, there is a maximum probability to detect three bursts from Wolf 424 . However, the papers about flares from dMe stars show that some stars which are further than others are more active and emit stronger bursts than other stars which are closer.

\section{Comparison between our results and the publications}

We have found no paper on the detection of bursts near $21 \mathrm{~cm}$ of TZ Ari, Gl. 569, V371 Ori and VW Com. The four other stars had been detected at $21 \mathrm{~cm}$ before the observations of 1989-1993: YY Gem and EQ Peg reached flux densities of only a few mJy, which is detectable by the VLA, not by the Arecibo telescope, and, to our knowledge, only Wolf 424 and YZ CMi were detected in Arecibo at $21 \mathrm{~cm}$ (see Sect. 3.3 in Abada-Simon et al. 1994), but they are not reported in the tables of Appendix 1 because precise information is lacking. In addition, these latter four stars emitted no burst during numerous hours of observations in Arecibo near $21 \mathrm{~cm}$, and these "negative" results have not been published (private communication). Table 3 gives a summary of the bursts noted down from the publications and a comparison with the 1989-1993 Arecibo campaign.

Using the results of the literature to determine the parameter a for each star, and assuming that the probability to detect $n$ bursts from one of the eight stars is estimated from Poisson's law, we deduce from Table 3 that during the 1989-1993 campaign in Arecibo we had a maximum probability $(36.6 \%)$ of detecting one burst from EQ Peg, but a probability almost as high (32.7\%) to detect no burst. On the other hand, we had the same probability $(22.5 \%)$ to detect two or three bursts from YZ CMi, and only $5.1 \%$ chances to detect no burst. We therefore "should have" detected one or several bursts from YZ CMi; but observations of this star with the VLA show that it emits bursts of weak amplitude (a few mJy) over long timescales (a few hours), which is difficult to detect with the Arecibo telescope. Finally, AD Leo had a very low probability (5.6\%) to emit the eleven bursts that we have actually detected. However, if the bursts detected in 1989-1993 actually correspond to less than eleven bursts, the probabilities inferred from the 1989-1993 campaign are then closer to those inferred from the literature. Concerning TZ Ari, Gl. 569, VW Com and V371 Ori, the published papers cannot allow to predict reliably the burst rate, since they report on less than half an hour of observation, which is not enough to establish significant statistics. Furthermore, Wolf 424 and YY Gem emitted no burst in 14 and 15.2 hours of reported observation, respectively: it is therefore not surprising that we detected nothing from them, but, on the other hand, the detection threshold published for Wolf 424 is much higher than that of the 1989-1993 campaign.

These Arecibo results can also be compared with some radio surveys of open clusters. Bastian et al. (1988) have used the VLA to search for radio emission from flare stars in the Pleiades: they detected no emission at $1.4 \mathrm{GHz}$ and their detection criteria led them to a frequency of "detectable" radio emitting events of $\leq 1$ event in 120 hours; they concluded that the flare stars in the Pleiades may be up to about ten times more active than flare stars 
Table 3. Comparison between the 1989-1993 campaign and former publications

\begin{tabular}{|c|c|c|c|c|}
\hline Stars & $\begin{array}{c}\text { Literature : } \\
\text { Number of } \\
\text { observing } \\
\text { hours at } 1.4 \\
\mathrm{GHz}\end{array}$ & $\begin{array}{c}\text { Number of } \\
\text { burst }\end{array}$ & $\begin{array}{c}1989-1993 \\
\text { campaign: } \\
\text { Number of } \\
\text { observing } \\
\text { hours at 1.4 } \\
\text { GHz }\end{array}$ & $\begin{array}{c}1989-1993 \\
\text { campaign: } \\
\text { Number of } \\
\text { bursts }\end{array}$ \\
\hline Wolf 424 & 14 & 0 & 10.7 & 0 \\
\hline TZ Ari & 0.4 & 0 & 3.3 & 0 \\
\hline AD Leo & 31 & 6 & 38.3 & 12 \\
\hline YZ CMi & 33.7 & $\geq 13$ & 7.7 & 0 \\
\hline EQ Peg & 16.1 & $>5$ & 3.6 & 0 \\
\hline GI 569 & 0.4 & 0 & 4.4 & 0 \\
\hline YY Gem & 15.2 & 0 & 3.1 & 0 \\
\hline VW Com & 0.4 & 0 & 2.0 & 0 \\
\hline
\end{tabular}

in the solar neighborhood, i.e. they are not very different. This seems to be confirmed if we put AD Leo at the Pleiades distance $(125 \mathrm{pc})$ : event its strongest burst detected in Arecibo in 1993 would be below the detection level of the VLA survey by Bastian et al. (1988). A survey of the Hyades made with the VLA at $1.5 \mathrm{GHz}$ by White et al. (1993) led to similar conclusions within several uncertainties: the Hyades $\mathrm{M}$ dwarfs are not much more active than the nearby population, and one can expect 1 event in 20 hours from the Hyades, located at 45 pc. At that distance, one or two of AD Leo's strongest bursts could have been detected in the 40 observing hours made in Arecibo between 1990 and 1993, which gives a rate of detectable events similar to the survey of White et al. (1993) or lower (for AD Leo's weaker bursts and for the other stars, not detected during the Arecibo campaign).

Let us now compare the activity rates in radio with those in the visible domain. There is a report of about 0.8 - 1.5 optical flares per hour from AD Leo (Pettersen et al. 1984; Pettersen et al. 1986; Pettersen et al. 1990), to compare with about 0.3 radio bursts detected per hour in 1989-1993, and with about 0.2 radio bursts per hour predicted from the literature. Doyle \& Mathioudakis (1990) estimate about 0.7 optical flares in $3 \mathrm{hr}$ from YY Gem, whereas no radio burst was detected in the same amount of time in 1989-1993; with the same optical rate of YY Gem, one expects about 3 optical flares in 15 hrs, during which no radio burst was found in the literature.

\section{Conclusion}

We have seen that our very reliable observing technique implies rates of dMe radio bursts per hour which are slightly different from what can be inferred from the "reliable" former papers. However, these rates may be underor over-estimated for various reasons. We have compared the stars with each other, but their rate of activity presumably depends on their instrinsic properties.
In reality, the peak flux density might not be the right indicator of rate of activity. In the visible domain, the activity rate is defined by Doyle \& Mathioudakis (1990) as the total energy released during a flare, divided by the total observing time. In order to calculate an equivalent quantity at radio wavelengths - although it might not be the best indicator - we need to know the total bandwidth of the emission, in addition to the distance of the source and duration of the burst. It is therefore not possible to estimate the total released energy from the papers. The energy of the bursts detected in 1989-1993 is of the order of $10^{17} \mathrm{~J}$, but it could be higher if the burst's bandwidth is much larger than the observing bandwidth.

Since dMe's flares are often compared to the Solar White Light Flares (WLF), it would also be interesting to compare the activity of dMe's in radio with the activity in the visible and X-rays. Indeed, the Solar WLF of type I are found to be correlated with X-rays and microwaves, whereas it is not the case for type II WLF. Although the last paragraph of Sect. 4 suggests that more optical flares than radio bursts are observed from flare stars, we point out that only numerous simultaneous observations can bring reliable statistical correlations between various wavelength domains: these are necessary to provide information on the triggering of flares and on the acceleration process(es). The correlation with other wavelengths and the rate of occurrence of each type of stellar radio burst is also necessary to understand the whole phenomenon of flares in dMe's.

Establishing reliable rates of activity for stars requires a large amount of observing hours. A long term study of dMe's radio bursts is necessary to establish the various types of radio bursts and the occurrence of each type. Collecting such data will be possible when the Arecibo and Nanay single-dish radiotelescopes are renovated. We have also extended these studies to decametric wavelengths, at which an array is easily accessible to us in Nanay (France): these observations are performed simultaneously with a 
complementary decametric array in Kharkov (Ukraine) and with an optical telescope in Nauchny (Crimea).

Acknowledgements. We are very grateful to N. Meyer-Vernet for encouraging this study. We thank H.J. Wendker for providing useful references, J. Aboudarham, T.S. Bastian, J.A. Bookbinder, S.M. White, and J. Lim for fruitful discussions, and the referee for suggesting improvements for the paper.

\section{References}

Abada-Simon M., Lecacheux A., Louarn P., et al., 1994, A\&A 288,219

Bastian T.S., Bookbinder J.A., 1987, Nat. 326, 678

Bastian T.S., Bookbinder J.A., Dulk G.A., Davis M., 1990, ApJ 353, 265

Bastian T.S., Dulk G.A., Slee O.B., 1988, ApJ 95, 794

Bondar N.I., 1995, A\&AS 111, 259

Doyle J.G., Mathioudakis M., 1990, A\&AS 227, 130

Gary D.E., 1985, in Radio Stars, Hjellming R.M. and Gibson D.M. (eds.) Astrophys. Space Sci. Library 116. Reidel

Güdel M., Benz A.O., Bastian T.S., et al., 1989, A\&A 220, L5

Güdel M., 1991, PhD Thesis, ETH Zürich

Haisch B.M., 1989, in IAU Colloq. 104: Solar and Stellar Flares, Haisch B.M. and Rodonò B.M. (eds.). Dordreicht: Kluwer Academic Publishers, reprinted from Solar Phys., 121, Nos. 1-2, p. 9

Jackson P.D., Kundu M.R., White S.M., 1989, A\&A 210, 284

Jackson P.D., Kundu M.R., Kassim N., 1990, Solar Phys. 130, 391

Jetsu L., 1993, A\&A 276, 345

Kundu M.R., Pallavicini R., White S.M., Jackson P.D., 1988, A\&A 195, 159

Kundu M.R., Shevgaonkar R.K., 1988, ApJ 334, 1001

Kundu M.R., White S.M., Jackson P.D., 1986, Adv. Spa. Res. 6,117

Lang K.R., Bookbinder J.A., Golub L., Davis M.M., 1983, ApJ 272, L15

Lang K.R., Willson R.F., 1986a, ApJ 302, L17

Lang K.R., Willson R.F., 1986b, ApJ 305, 363

Lang K.R., Willson R.F., 1988, ApJ 326, 300

Lecacheux A., Abada-Simon M., Belkora L., et al., 1992, in Seventh Cambridge Workshop on Cool Stars, Stellar Systems, and the Sun, Giampapa S. and Bookbinder J.A. (eds.). ASP Conf. Ser. 26, 319

Lecacheux A., Rosolen C., Davis M., et al., 1993, A\&A 275, 670
Lefèvre E., Klein K.L., Lestrade J.F., 1994, A\&A 283, 483 Moffet J.T., Helmken H.F., Spangler S.R., 1978, PASP 90, 93 Petit M., 1987, Variable Stars

Pettersen B.R., Coleman L.A., Evans D.S., 1984, ApJS 54, 375 Pettersen B.R., Panov K.P., Sandmann W.H., Ivanova M.S., 1986, A\&AS 66, 235

Pettersen B.R., Panov K.P., Ivanova M.S., et al., 1990, in Flare Stars in Star Clusters, Associations and the Solar Vicinity, IAU 137, Mirzoyan L.V., Pettersen B.R. and Tsvetkov M.K. (eds.). Kluwer Academic Publishers

Phillips M.J., Hartmann L., 1978, ApJ 224, 182

White S.M., Kundu M.R., Jackson P.D., 1986, ApJ 311, 814

White S.M., Jackson P.D., Kundu M.R., 1989, ApJS 71, 895

White S.M., Jackson P.D., Kundu M.R., 1993, ApJ 105, 563

Willson R.F., Lang K.R., Foster P., 1988, 199, 255

\section{Appendix 1}

\section{Number of radio bursts detected from the nine studied dMe flare stars}

The tables report:

- Column 1: "N(h)": total number of observing hours near $21 \mathrm{~cm}$,

- Column 2: "n": total number of detected bursts,

- Column 3: " $\Delta t$ ": duration of each burst,

- Column 4: "I $I_{\mathrm{Max}}(\mathrm{mJy})$ ": peak flux density (shortest reported value, either for a 1-s integration time or with a shorter time resolution),

- Column 5: "Circ. Polar.": Rate of circular polarisation (" $R$ " for right and " $L$ " for left),

- Column 6: "Quiesc. (mJy)": Flux density of the quiescent emission possibly detected,

- Column 7: Observing instrument,

- Column 8: Reference(s).

In each of the tables below, we firstly report the number of bursts detected with one of the three techniques mentioned in Sect. 2.1. Below the total of bursts detected according to these criteria, we also mention some bursts whose stellar origin is not perfectly established. 


\section{Scheme of the table's structure}

\begin{tabular}{|l|}
\hline VLA and other reliable interferometers \\
\hline Several "single-dish" radiotelescopes observing simultaneously: \\
Arecibo (Puerto-Rico), Effelsberg (Germany) and Jodrell Bank (United Kingdom) \\
\hline Reliable "ON-OFF" with a single dish instrument \\
\hline \hline Total \\
\hline \hline "Single-dish" radiotelescope without any of the "reliable" criteria \\
Less reliable observations \\
\hline
\end{tabular}

\section{AD Leo}

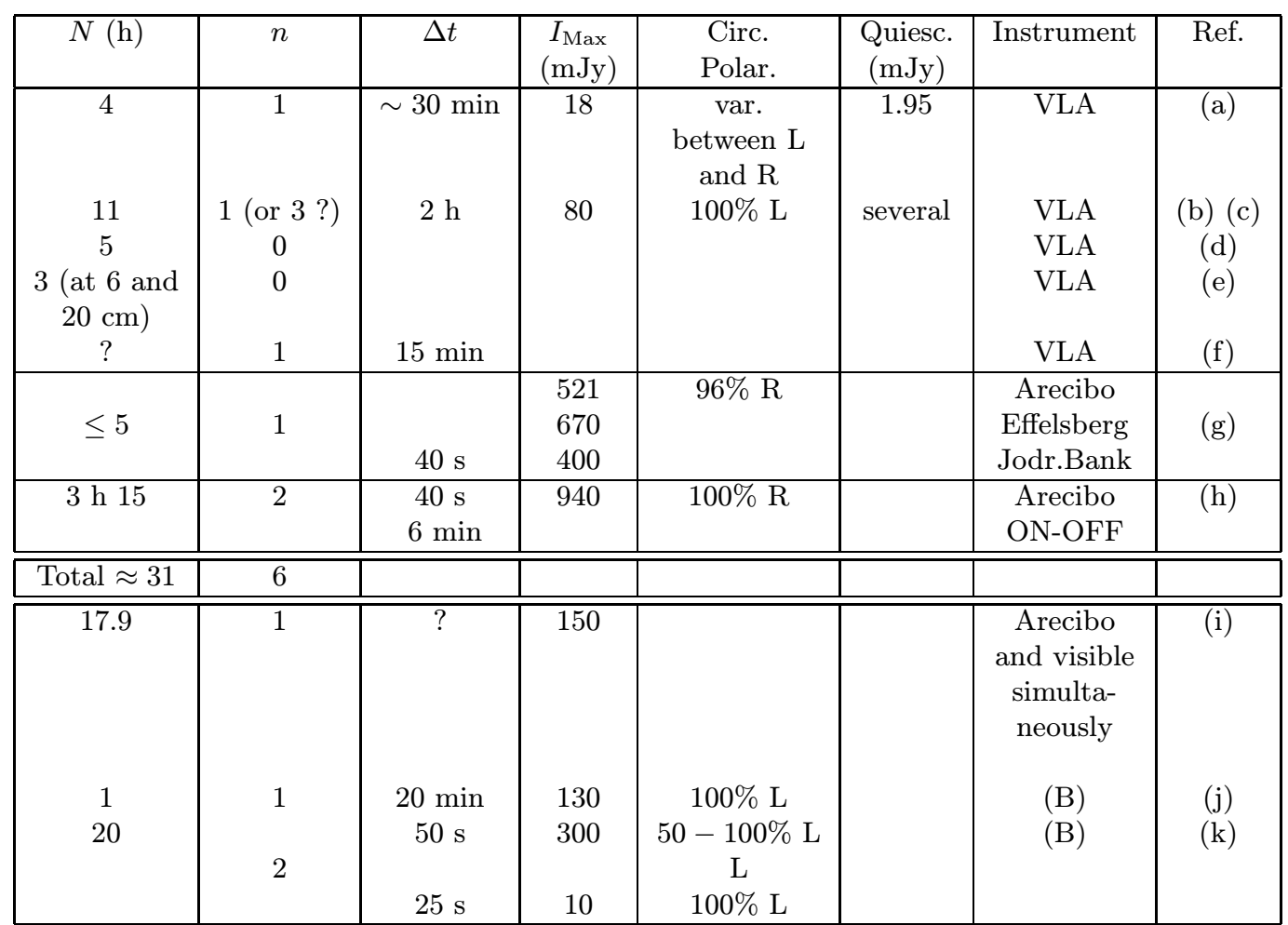

Notes:

(A) Simultaneous observations in Arecibo and in optical within a few seconds, but the authors do not believe this detection! (B) Observation in Arecibo, but without guarantee on the stellar origin.

\section{References:}
(a) Jackson et al. (1989)
(b) Kundu \& Shevgaonkar (1988)
(c) Kundu et al. (1986)
(d) Bastian \& Bookbinder (1987)
(e) Willson et al. (1988)
(f) Jackson et al. (1990)
(g) Güdel et al. (1989)
(h) Bastian et al. (1990)
(i) Moffett et al. (1978)
(j) Lang et al. (1983)
(k) Lang \& Willson (1986b). 


\section{EQ Peg}

\begin{tabular}{|c|c|c|c|c|c|c|c|}
\hline$N(\mathrm{~h})$ & $n$ & $\overline{\Delta t}$ & $\begin{array}{l}I_{\mathrm{Max}} \\
(\mathrm{mJy})\end{array}$ & $\begin{array}{c}\text { Circ. } \\
\text { Polar. }\end{array}$ & $\begin{array}{c}\text { Quiesc. } \\
\text { (mJy) }\end{array}$ & Instrument & Ref. \\
\hline 3.75 & $\begin{array}{l}\text { "nume } \\
\text { rous" }\end{array}$ & $\begin{array}{l}<30 \text { min } \\
\text { (chacun) }\end{array}$ & $\sim 27$ & high & & VLA & (a) \\
\hline 0.5 & 1 & $10 \mathrm{~min}$ & $\begin{array}{c}8.4 \\
9\end{array}$ & ? R & 1.5 & VLA & (b) \\
\hline 5 & 3 & $\begin{array}{l}10 \mathrm{~min} \\
2 \mathrm{~h}\end{array}$ & $\begin{array}{l}12 \\
45\end{array}$ & $\begin{array}{c}30-60 \% \mathrm{R} \\
\mathrm{R} \\
10-80 \% \\
\mathrm{R}\end{array}$ & & VLA & (c) \\
\hline $\begin{array}{r}2.57 \\
4.28 \\
\end{array}$ & $\begin{array}{l}0 \\
0 \\
\end{array}$ & & & & & $\begin{array}{c}\text { Jodrell } \\
\text { Bank } \\
\text { Effelsberg } \\
\end{array}$ & $\begin{array}{l}\text { (d) } \\
\text { (d) }\end{array}$ \\
\hline $\begin{array}{l}\text { Total } \\
\approx 16.1 \\
\end{array}$ & $>5$ & & & & & & \\
\hline$?$ & 0 & & & & 0.47 & VLA & $(\mathrm{e})$ \\
\hline
\end{tabular}

(a) Kundu et al. (1986)

(b) Kundu et al. (1988)

(c) Bastian \& Bookbinder (1987)

(d) Güdel (1991)

(e) Jackson et al. (1989).

Wolf 424

\begin{tabular}{|c|c|c|c|c|c|c|c|}
\hline$N(\mathrm{~h})$ & $n$ & $\Delta t$ & $\begin{array}{c}I_{\text {Max }} \\
(\mathrm{mJy})\end{array}$ & $\begin{array}{c}\text { Circ. } \\
\text { Polar. }\end{array}$ & $\begin{array}{c}\text { Quiesc. } \\
(\mathrm{mJy})\end{array}$ & Instrument & Ref. \\
\hline 0.4 & $\begin{array}{c}0(\mathrm{no} \\
13.6\end{array}$ & $\begin{array}{c}\text { burst }>100 \\
\mathrm{mJy})\end{array}$ & & & 0.4 & $\begin{array}{c}\text { VLA } \\
\text { Arecibo } \\
\text { and visible } \\
\text { simulta- } \\
\text { neou sly }\end{array}$ & $\begin{array}{c}(\mathrm{b}) \\
\text { mJy) }\end{array}$ \\
\hline \hline Total $\approx 14$ & 0 & & & & & & \\
\hline \hline
\end{tabular}

(a) White et al. (1989)

(b) Moffett et al. (1978). 
YZ CMi

\begin{tabular}{|c|c|c|c|c|c|c|c|}
\hline$N(\mathrm{~h})$ & $n$ & $\Delta t$ & $\begin{array}{l}I_{\mathrm{Max}} \\
(\mathrm{mJy})\end{array}$ & $\begin{array}{l}\text { Circ. } \\
\text { Polar. }\end{array}$ & $\begin{array}{c}\text { Quiesc. } \\
\text { (mJy) }\end{array}$ & Instrument & Ref. \\
\hline 4.5 & $\begin{array}{c}\text { slowly } \\
\text { variable } \\
\text { emission }\end{array}$ & & 30 & $\begin{array}{c}\text { variable: } \\
35 \%- \\
90 \% \mathrm{~L}\end{array}$ & & VLA & (a) \\
\hline $4 \mathrm{~h} 12$ & $\begin{array}{l}8 \text { (one } \\
\text { after the } \\
\text { others) }\end{array}$ & $\sim 30 \mathrm{~min}$ & 16 & $\sim 100 \% \mathrm{~L}$ & & VLA & (b) \\
\hline 3 & 1 & $\sim 20 \mathrm{~min}$ & 15 & 0 & & VLA & (c) \\
\hline $\begin{array}{c}8 \text { (at } 20 \\
\text { and } 90 \\
\mathrm{~cm})\end{array}$ & & $\sim 8 \mathrm{~h}$ & 9.2 & $94 \% \mathrm{~L}$ & 4.7 & VLA & (d) \\
\hline $\begin{array}{l}5 \\
9\end{array}$ & $\begin{array}{l}0 \\
1\end{array}$ & $\sim 5 \mathrm{~h}$ & 15 & $100 \% \mathrm{~L}$ & 2 & VLA & $\begin{array}{l}\text { (f) }(\mathrm{g}) \\
\text { (h) }\end{array}$ \\
\hline $\begin{array}{c}\text { Total } \approx \\
33.7\end{array}$ & $>13$ & & & & & & \\
\hline $\begin{array}{c}? \\
? \\
2.4\end{array}$ & $\begin{array}{l}1 \\
1 \\
1\end{array}$ & $\begin{array}{c}2 \mathrm{~h} 20 \\
2 \mathrm{~h} \\
40 \mathrm{~s}\end{array}$ & $\begin{array}{c}3.9 \\
5 \\
150\end{array}$ & $80 \% \mathrm{~L}$ & 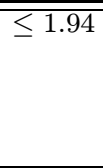 & $\begin{array}{c}\text { VLA } \\
\text { VLA } \\
\text { Arecibo } \\
\text { and visible } \\
\text { (C) }\end{array}$ & $\begin{array}{l}\text { (i) } \\
\text { (g) } \\
\text { (j) }\end{array}$ \\
\hline
\end{tabular}

Note: (C) The radio peak was detected 30 s before the optical: is it reliable?
(a) Lang \& Willson (1986a)
(b) Lang \& Willson (1988)
(c) Willson et al. (1988)
(d) Kundu \& Shevgaonkar (1988)
(e) Bastian \& Bookbinder (1987)
(f) Kundu et al. (1986)
(g) Jackson et al. (1990)
(h) Kundu \& White (1989)
(j) Moffett et al. (1978).

YY Gem

\begin{tabular}{|c|c|c|c|c|c|c|c|}
\hline$N(\mathrm{~h})$ & $n$ & $\Delta t$ & $\begin{array}{c}I_{\text {Max }} \\
(\mathrm{mJy})\end{array}$ & $\begin{array}{c}\text { Circ. } \\
\text { Polar. }\end{array}$ & $\begin{array}{c}\text { Quiesc. } \\
(\mathrm{mJy})\end{array}$ & Instrument & Ref. \\
\hline $\begin{array}{c}1 \mathrm{~h} 13 \\
14 \mathrm{~h}\end{array}$ & 0 & & & & 0.41 & VLA & $(\mathrm{a})$ \\
$(\mathrm{b})$
\end{tabular}

(a) Jackson et al. (1989)

(b) Gary (1985).

TZ Ari

\begin{tabular}{|c|c|c|c|c|c|c|c|}
\hline$N(\mathrm{~h})$ & $n$ & $\Delta t$ & $\begin{array}{c}I_{\text {Max }} \\
(\mathrm{mJy})\end{array}$ & $\begin{array}{c}\text { Circ. } \\
\text { Polar. }\end{array}$ & $\begin{array}{c}\text { Quiesc. } \\
(\mathrm{mJy})\end{array}$ & Instrument & Ref. \\
\hline 0.4 & $\begin{array}{c}0 \\
(<0.48 \\
\mathrm{mJy})\end{array}$ & & & & & VLA & $(\mathrm{a})$ \\
\hline
\end{tabular}

(a) White et al. (1989). 
G1 569

\begin{tabular}{|c|c|c|c|c|c|c|c|}
\hline$N(\mathrm{~h})$ & $n$ & $\Delta t$ & $\begin{array}{c}I_{\text {Max }} \\
(\mathrm{mJy})\end{array}$ & $\begin{array}{c}\text { Circ. } \\
\text { Polar. }\end{array}$ & $\begin{array}{c}\text { Quiesc. } \\
(\mathrm{mJy})\end{array}$ & Instrument & Ref. \\
\hline 0.4 & $\begin{array}{c}0 \\
(<0.42 \\
\mathrm{mJy})\end{array}$ & & & & & VLA & $(\mathrm{a})$ \\
\hline
\end{tabular}

a) White et al. (1989).

VW Com

\begin{tabular}{|c|c|c|c|c|c|c|c|}
\hline$N(\mathrm{~h})$ & $n$ & $\Delta t$ & $\begin{array}{c}I_{\text {Max }} \\
(\mathrm{mJy})\end{array}$ & $\begin{array}{c}\text { Circ. } \\
\text { Polar. }\end{array}$ & $\begin{array}{c}\text { Quiesc. } \\
(\mathrm{mJy})\end{array}$ & Instrument & Ref. \\
\hline 0.4 & $\begin{array}{c}0 \\
(<0.58 \\
\mathrm{mJy})\end{array}$ & & & & & VLA & $(\mathrm{a})$ \\
\hline
\end{tabular}

(a) White et al. (1989). 